\title{
Limb Development
}

National Cancer Institute

\section{Source}

National Cancer Institute. Limb Development. NCI Thesaurus. Code C19946.

Interdependent positional signaling by the apical ectodermal ridge, the posterior

mesenchyme zone of polarizing activity, and the non ridge ectoderm coordinate limb bud outgrowth and patterning, Limb Development, by migrated lateral plate mesoderm and somite cells. Proximal-distal differentiating mesenchymal cells condense to form the limb skeletal primordia. 